\title{
Atrophodermia linearis Moulin
}

\author{
Linear Atrophoderma of Moulin
}

Autoren

Institut

\section{Palle, C. Bayerl}

Klinik für Dermatologie und Allergologie, Städtisches Klinikum Wiesbaden, HSK Wilhelm-Fresenius-Klinik GmbH Lehrkrankenhaus der Universität Mainz

\section{Bibliografie}

DOI $10.1055 / s-2008-1077629$

Online-Publikation: 13.1.2009

Akt Dermatol 2009; 35:

46-48 @ Georg Thieme

Verlag KG Stuttgart · New York ISSN 0340-2541

Korrespondenzadresse

\section{Markus Palle}

Klinik für Dermatologie und Allergologie

Städtisches Klinikum Wiesbaden HSK, Wilhelm-Fresenius-Klinik $\mathrm{GmbH}$

Aukammallee 39

65191 Wiesbaden

Markus.Palle@hsk-wiesbaden.de

\section{Zusammenfassung \\ $\nabla$}

Atrophodermia linearis Moulin (ALM) ist eine unverwechselbare Krankheit, die erstmals 1992 von Moulin et al. beschrieben wurde [8]. Sie zeichnet sich durch leicht atrophische, nicht sklerosierte, hyperpigmentierte bandähnliche Hautläsionen aus, welche vorwiegend am Stamm lokalisiert sind und den Blaschko-Linien folgen.

\section{Krankengeschichte}

$\nabla$

Eine 32-jährige Patientin stellte sich in unserer Ambulanz mit asymptomatischen, leicht atrophischen, hyperpigmentierten, stammbetonten, bandförmigen Hautveränderungen vor.

Die Hautveränderungen traten um das 12. Lebensjahr auf. Sie waren weder juckend noch schmerzhaft, noch waren eine vorangegangene Entzündung, ein Erythem oder Bläschen erinnerlich. Seit dieser Zeit trat auch keine Progression oder Regression der Maculae auf. Die Hautstrukturen und der Haarwuchs zeigten sich normal. Es fand sich kein Hinweis auf eine Muskelatrophie, weiter zeigte sich das zentrale Nervensystem und das Skelettsystem unauffällig. Die Patientin nahm keine Medikamente ein und es bestanden auch keine Atopie oder Allergien. Die Familienanamnese zeigte sich hinsichtlich von Hauterkrankungen negativ. Nur in der Blutuntersuchung der Patientin war eine Erhöhung der antinukleären Antikörper von 1:640 aufgefallen.

\section{Hautbefund \\ $\nabla$}

Am rechten Abdomen fanden sich scharf zur Mittellinie hin begrenzt, flächige, hellbraune, nicht indurierte, teilweise etwas unter dem Hautniveau liegende, z. T. streifige, den BlaschkoLinien folgende Maculae ( $\bullet$ Abb. 1 - 3). Auch am
Eine vorangegangene Entzündung besteht nicht. Die Erkrankung tritt im Kindes- oder Jugendalter auf und hat einen chronischen Verlauf ohne Progression oder Regression. In der Literatur sind ca. 25 Fälle beschrieben worden [4]. Wir berichten über eine 32-jährige Patientin mit einer seit 20 Jahren bestehenden Atrophodermia, welche den Blaschko-Linien folgt und auf den Stamm und den linken Arm beschränkt ist.

Dekolleté, der linken Brust und am oberen Rücken bestanden münzgroße teils ovale, konfluierende z.T. unregelmäßig geformte, unscharf begrenzte Maculae. Am linken Arm zeigte sich ein einige $\mathrm{cm}$ breites, unregelmäßig begrenztes, linienförmiges hyperpigmentiertes Band, welches sich von der linken Brust bis zum distalen Unterarm erstreckte ( $\bullet$ Abb. 4). Epiluminiszenzmikroskopisch fand sich in den Läsionen kein Pigmentnetz.

\section{Laborchemische Untersuchungen $\nabla$}

Im Routinelabor zeigte sich nur die Harnsäure ein wenig erhöht, die übrigen Werte der klinischen Chemie sowie das Blutbild waren unauffällig. Die Borrelien-Serologie sowie die ENA und dsDNS-Antikörper waren negativ. Lediglich die antinukleären Antikörper waren positiv (jetzt $1: 800)$.

\section{Apparative Untersuchungen \\ $\nabla$}

Im Röntgen-Thorax fand sich ein altersentsprechender regelrechter kardio-pulmonaler Befund. In der Lungenfunktionstestung war keine Ventilationsstörung feststellbar.

In der Abdomen-Sonografie zeigte sich kein pathologischer Befund der intraabdominellen 


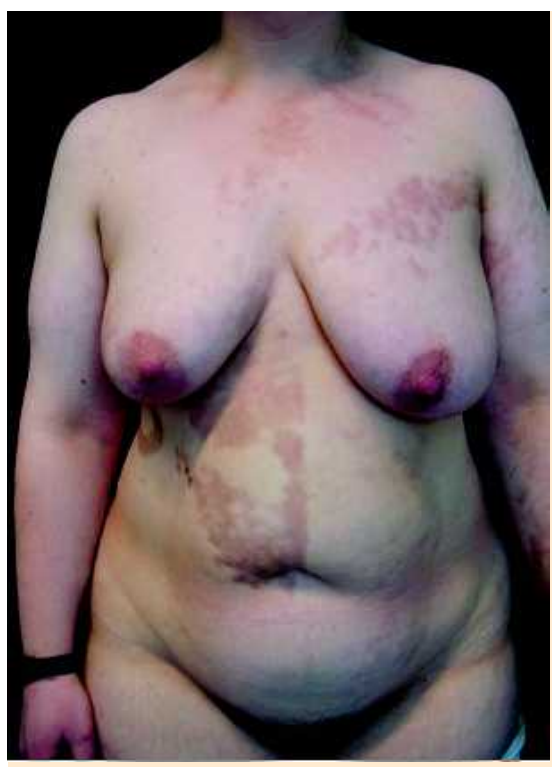

Abb. 1 Scharfe Abgrenzung zur Mittellinie.

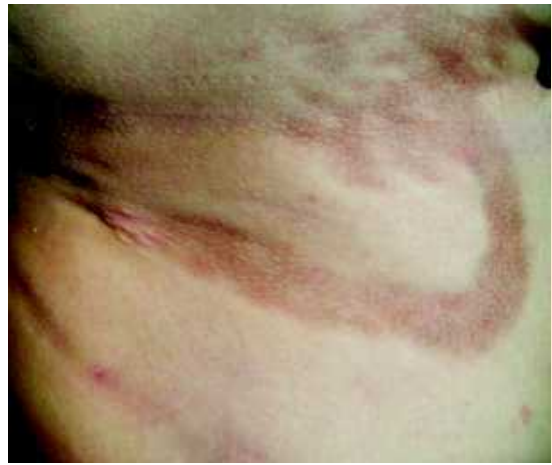

Abb. 2 Ausbreitung entlang der BlaschkoLinien.

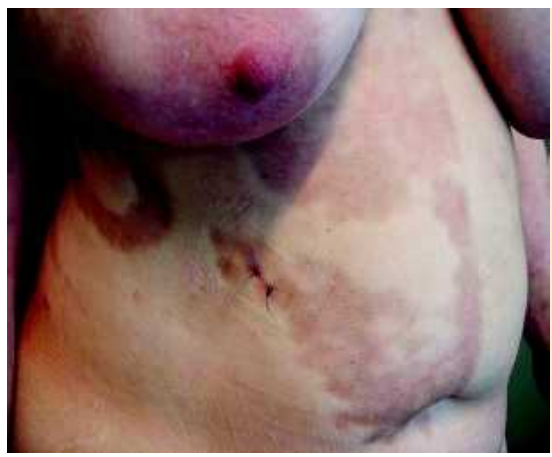

Abb. 3 Bandförmige Macula an der rechten Flanke.

Organe, auch die Ösophagus-Funktionsszintigrafie war unauffällig.

Im EKG fand sich ein Sinusrhythmus, eine Herzfrequenz von 83/min, keine Erregungsrückbildungsstörungen, sowie in der Echokardiografie ein Normalbefund.

Wir führten eine Biopsie der Haut am rechten Oberbauch durch. Der Immunfluoreszenz-histologische Befundbericht war negativ, auch fand sich in der Elastica-Färbung kein pathologischer Befund, die elastischen Fasern zeigten sich unauffällig. In der oberen und mittleren Dermis bestand ein diskretes perivaskuläres lymphohistiozytäres Infiltrat. Weiter fand sich in den Basalschichten der Epidermis eine geringe Melaninvermehrung.

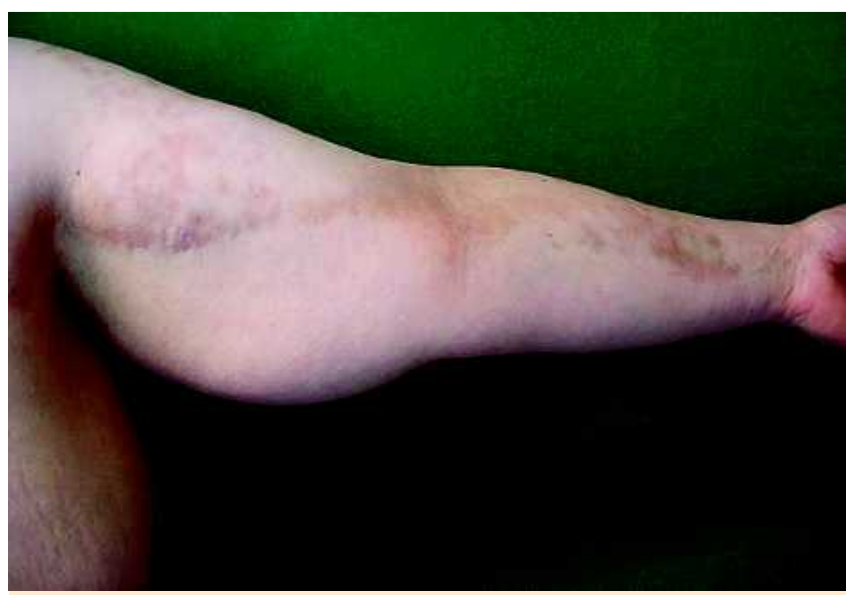

Abb. 4 Linienförmige Ausbreitung am linken Arm.

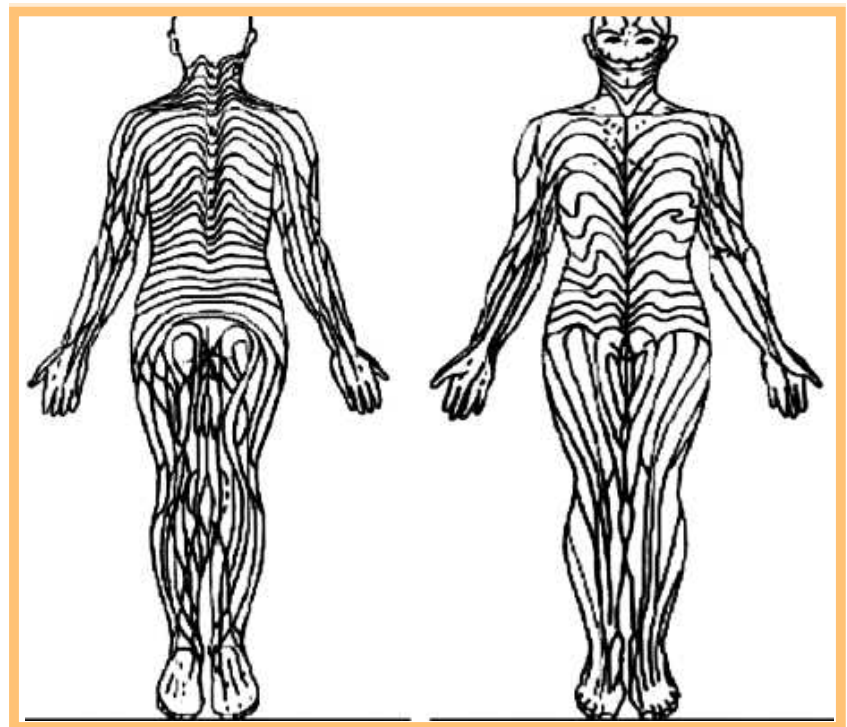

Abb. 5 Anordnung der Blaschko-Linien.

\section{Diskussion}

$\nabla$

Atrophodermia linearis Moulin hat wegen der geringen Atrophie und der Hyperpigmentierung eine starke Ähnlichkeit mit der Atrophodermia ideopathica progressiva Pasini et Pierini. Jedoch folgt die ALM den Blaschko-Linien und wurde erstmals 1992 von Moulin nach der Untersuchung von 5 Patienten beschrieben [8]. Die Blaschko-Linien korrelieren weder mit neuralen noch mit vaskulären Versorgungsgebieten, sondern entsprechen vermutlich embryonalen Wachstumslinien der Haut $[7,9,10]$.

Diese wurden erstmals vom Berliner Dermatologen Alfred Blaschko (1858-1922) im Jahre 1901 beschrieben ( $\bullet$ Abb. 5) [1].

Die Blaschko-Linien müssen von Dermatomen und Hautspaltlinien unterschieden werden. Nach dem klinischen Bild und der Pathogenese kann man die den Blaschko-Linien folgenden Dermatosen in 3 Untergruppen gliedern ( $\bullet$ Tab. 1 - 3) [3].

Die Ätiologie der ALM ist ein genetischer Mosaizismus, der durch eine somatische Mutation einzelner somatischer Zelllinien (die im Unterschied zu den Keimbahnmutationen nicht vererbt 
Tab. 1 Genodermatosen, die den Blaschko-Linien folgen.

Incontinentia pigmentii
Fokale dermale Hypoplasie
Menkes- Syndrom
Hidrotische ektodermale Dysplasie (x-chromosomal)
Conradi-Hünermann-Syndrom
CHILD-Syndrom
Oro-facial-digital-Syndrom, Typ I
Eschenblatt-Hypomelanose (,ash-leaf-Macula“)
Familiäre kutane Amyloidose, Partington-Typ
Café-au-lait-Fleck beim McCune-Albright-Syndrom

Tab.2 Angeborene und/oder nävoide Erkrankungen, die den BlaschkoLinien folgen.

\begin{tabular}{l} 
Hypomelanose Ito \\
Nävus depigmentosus \\
Lineare nävoide Hypermelanose \\
Nävus sebaceous Jadassohn \\
Epidermaler Nävus \\
Lineare epidermolytische Hyperkeratose \\
\hline Entzündlicher linearer verruköser epidermaler Nävus \\
Porokeratose Mibelli \\
Nävus comedonicus \\
Nävus corniculatus \\
Morbus Darier \\
\hline Transiente akantholytische Dermatose \\
Ekkrine Nävi \\
Syringozystadenoma papilliferum \\
Basalzellnävussyndrom \\
Unilaterales nävoides Basalzellkarzinom \\
Bart-Syndrom
\end{tabular}

Tab. 3 Erworbene Dermatosen, die den Blaschko-Linien folgen.

Lichen striatus
Lineare Psoriasis
Linearer Lichen planus
Lineare Muzinose und Mycosis fungoides
Segmentale Vitiligo
Lineares fixes Arzneimittelexanthem
Lupus erythematosus (LE)
(linearer kutaner LE und linearer LE profundus)
Extragenitaler Lichen sklerosus
Generalisiertes lichenoides Arzneimittelexanthem
Atrophodermia linearis Moulin
Lineare Sklerodermie

wird) in der Embryogenese zustande kommt [5,9,11]. Die ALM tritt sowohl bei männlichen als auch bei weiblichen Personen auf, wobei jedoch Frauen häufiger betroffen sind [6]. Ca. 25 Fälle der ALM wurden bisher in der Literatur beschrieben [4]. Im Jahre 1994 stellten Baumann et al. einen weiteren Fall eines Patienten mit ALM vor [1]. Dieser Fall unterschied sich von denen von Moulin et al. durch den Nachweis von antinukleären Antikörpern, wie dies auch bei unserer Patientin der Fall war.

Weder zeigte sich bei unserer Patientin eine entzündliche Randzone noch die Ausbildung eines Erythems. Des Weiteren fand sich trotz 20-jährigem Bestehen der Hautläsionen keine Muskelatrophie und auch keine Knochenveränderungen. Auffällig war wie bei Baumann et al. eine Erhöhung der antinukleären Antikörper [1]. Diese Erhöhung wurde bei den Fällen von Moulin et al. nicht nachgewiesen. Jedoch beschrieben Wokalek et al. im Jahre 1985 eine Atrophodermia idiopathica progressiva Pasini et Pierini in zosteriformer Anordnung mit Nachweis von antinukleären Antikörpern. Hierbei handelte es sich höchstwahrscheinlich auch um eine ALM [2].

In unserem Fall konnte aufgrund der ausführlichen differenzialdiagnostischen Untersuchungen eine andere streifenförmige Erkrankung ausgeschlossen werden.

Hinsichtlich der Therapie ist zu sagen, dass in der Studie bei Baumann et al. durch topische Anwendung von Steroiden und Heparin sowie in der Studie bei Wollenberg et al. und Rompel et al., welche hochdosiert Penicillin intravenös verordneten, kein positiver Effekt erzielt werden konnte [2].

Abschließend ist zu sagen, dass zur Zeit keine wirksame Behandlung der ALM existiert. Diese Situation ist sehr belastend für Patienten, da die ALM oft eine erhebliche Beeinträchtigung der Lebenssituation darstellt und zu erheblichem emotionalen Stress führen kann.

\section{Abstract}

\section{Linear Atrophoderma of Moulin $\nabla$}

Linear atrophoderma of Moulin is a distinctive disease originally described by Moulin et al. in the year 1992 and characterized by mildly atrophic, non-sclerotic, hyperpigmented band-like lesions mostly localised on the trunk following the lines of Blaschko. No preceeding inflammation is noted. Usually, the condition begins in childhood or adolescence. The disease is chronic without progression or regression. Here we describe a 32-year-old female with a 20 year history of a linear atrophoderma of Moulin following the lines of Blaschko that involved the trunk and the left arm.

\section{Literatur}

1 Baumann L, Happle R, Plewig G, Schirren CG. Atrophodermia linearis Moulin. Ein neues Krankheitsbild, das den Blaschko-Linien folgt. Hautarzt 1994; 45: $231-236$

2 Utikal J, Keil D, Klemke CD, Bayerl C, Goerdt S. Predominant telangiectatic erythema in linear atrophoderma of Moulin. novel variant or separate entity? Dermatology 2003; 207: 310-315

3 Tagra S, Talwar AK, Walia RS. Lines of Blaschko. Indian Journal of Dermatology, Venereologie and Leprology 2005; 71: 57-59

4 Cecchi R, Bartoli L, Brunetti L, Pavesi M. Linear atrophoderma of Moulin localised on the neck. Dermatology online journal 2008; 14 (6): 12

5 Rompel R, Mischke AL, Langner C, Happle R. Linear atrophoderma of Moulin. European Journal of Dermatology 2000; 10: 611 -613

6 Miteva L, Nikolova K, Obreshkova E. Linear atrophoderma of Moulin. International Journal of Dermatology 2004; 44: 867-869

7 Peching G, Galarza C, Kumakawa Z, Mendoza D, Morante V. Atrofodermia que sigue líneas de Blaschko. Atrophoderma that follows Blaschko's lines. Dermatology Peru 2005; 15: 66- 69

8 Moulin G, Hill MP, Guillaud V et al. Acquired atrophic pigmented bandlike lesions following laschko's lines. Ann Dermatol Venereol 1992; 119: $729-736$

9 Höger P. Klinik für Dermatologie der Universität Hamburg. BlaschkoLinien: "Skizzen" aus dem Mutterleib. Hautnah Dermatologie 2003; 2:

10 Wollenberg A, Baumann L, Plewig G. Linear atrophoderma of Moulin: a disease which follows Blaschko's lines. Br J Dermatol 1996; 135: 277 279

11 Danarti R, Bittar M, Happle R, König A. Linear atrophoderma of Moulin. Postulation of mosaicism for a predisposing gene. J Am Acad Dermatol 2003; 49: $492-498$ 